\title{
Enhancement and Quenching of Fluorescence by Silver Nanoparticles in Organic Light-Emitting Diodes
}

\author{
Ying-Chung Chen, ${ }^{1}$ Chia-Yuan Gao, ${ }^{1}$ Kan-Lin Chen, ${ }^{2}$ \\ Teen-Hang Meen, ${ }^{3}$ and Chien-Jung Huang ${ }^{4}$ \\ ${ }^{1}$ Department of Electrical Engineering, National Sun Yat-sen University, Kaohsiung 80424, Taiwan \\ ${ }^{2}$ Department of Electronic Engineering, Fortune Institute of Technology, Kaohsiung 83160, Taiwan \\ ${ }^{3}$ Department of Electronic Engineering, National Formosa University, Hu-Wei, Yunlin 63201, Taiwan \\ ${ }^{4}$ Department of Applied Physics, National University of Kaohsiung, Kaohsiung 81148, Taiwan
}

Correspondence should be addressed to Kan-Lin Chen; klchen@fotech.edu.tw and Chien-Jung Huang; chien@nuk.edu.tw

Received 8 November 2013; Accepted 14 November 2013

Academic Editor: Liang-Wen Ji

Copyright (C) 2013 Ying-Chung Chen et al. This is an open access article distributed under the Creative Commons Attribution License, which permits unrestricted use, distribution, and reproduction in any medium, provided the original work is properly cited.

\begin{abstract}
The influence of silver nanoparticles (SNPs) on the performance of organic light-emitting diodes (OLEDs) is investigated in this study. The SNPs are introduced between the electron-transport layers by means of thermal evaporation. SNPs are found to have the surface plasmon resonance at wavelength $525 \mathrm{~nm}$ when the mean particle size of SNPs is $34 \mathrm{~nm}$. The optimized OLED, in terms of the spacing between the emitting layer and SNPs, is found to have the maximum luminance 2.4 times higher than that in the OLED without SNPs. The energy transfer between exciton and surface plasmons with the different spacing distances has been studied.
\end{abstract}

\section{Introduction}

The organic light-emitting diode (OLED) has displayed significant potentiality in the applications of flat panel display and lighting, which is mainly because of its low power consumption. Various means have been developed to optimize OLED's efficiency, for example, doping the hole-transporting materials or inserting the hole-blocking layer to decrease hole mobility and balance hole/electrons ratio in emitting layer (EML) of device [1-3], doping phosphorescence material into the EML to increase the quantum efficiency by taking advantage of triplet state recombination [4-6], or improving the hole/electron balance by increasing electron injection $[7,8]$. However, those methods increased the complexity of device structure which increases the fabrication time and cost of device.

Recently, in view of the luminance enhancement effect of surface plasmon (SP), the surface plasmon resonance effect (SPRE) of metal nanoparticles has been actively studied in both OLED and inorganic light-emitting diode in [9-12]. It is well known that the SPRE was induced by interaction between the surface charge of metal and the electromagnetic field of the incident light [13]. When the metal surface is close to the fluorescent molecule, the luminous efficiency of the fluorescent molecule can be significantly enhanced. Nevertheless, the nonradiative quenching of exciton at the metal surface occurs when the distance is much smaller between the fluorescent molecule and the metal surface. Therefore, two competitive processes take place in an excitonSP system: (i) increase in radiative intensity by subtraction in decay time of exciton and (ii) nonradiative quenching of exciton by energy transfer to the metal surface. Therefore, the distances between the fluorescent molecule and the metal surface are a very important parameter in terms of the coupling between the SPs and excitons.

The silver nanoparticles (SNPs) layer was incorporated as an interlayer between the electron-transport layer and the electrode, which can improve the efficiency of OLEDs [14]. Nevertheless, the causes of nonradiative quenching and efficiency improvement at different distances between the silver nanoparticles and electron-transport layer were not described in detail. In this paper, the distance of the SNPs 


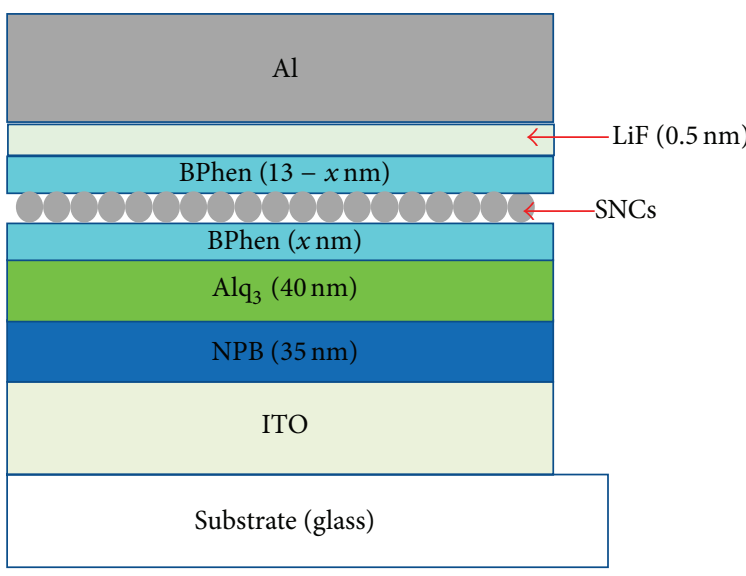

FIgURE 1: Schematic structure of OLED with different spacing distances between EML and SNPs.

from the EML has been optimized for enhancement of maximum luminescence, and how the distances between the SNPs and the EML influenced the enhancement of device luminescence was also observed. Furthermore, mechanisms of increase in radiative intensity and nonradiative quenching have been described in detail.

\section{Experimental}

The schematic structure of OLEDs is shown in Figure 1. Indium tin oxide (ITO) coated on glass with a sheet resistance of $10 \Omega /$ sq was used as the starting substrate. The substrate was cleaned with acetone, methanol, and deionized water and then dried with nitrogen gas. After the cleaning process, the substrates were loaded into a thermal evaporator. Afterward, N,N'-bis-(1-naphthyl)-N,N-diphenyl-1,1' -biphenyl1-4diamine (NPB; $35 \mathrm{~nm}$ ), tris-(8-hydroxyquinoline) aluminum $\left(\mathrm{Alq}_{3} ; 40 \mathrm{~nm}\right), 4,7$-diphenyl-1-1,10-phenanthroline (BPhen; $\mathrm{Xnm}$ ), SNPs, BPhen (13-X nm), lithium fluoride (LiF; $0.5 \mathrm{~nm}$ ), and aluminum ( $\mathrm{Al} ; 100 \mathrm{~nm})$ were deposited. NPB and $\mathrm{BPhen}$ were used as the hole and electron-transport layers, respectively. $\mathrm{Alq}_{3}$ has been used as EML. $\mathrm{LiF}, \mathrm{Al}$, and ITO have been used as the electron-injection layer, cathode, and anode, respectively. In addition, the SNCs were fabricated by thermal evaporation of silver slug. The deposition rate and the deposition time are $0.01 \mathrm{~nm} / \mathrm{s}$ and 300 seconds. During the deposition, the base pressure of the chamber was maintained at as low as $2.4 \times 10^{-6}$ Torr. The active area of the device was $6 \times 6 \mathrm{~mm}^{2}$. The deposition rates of all organic materials were maintained at $0.01 \mathrm{~nm} / \mathrm{s}$, except for the $\mathrm{Al}$ whose deposition rate was $0.5 \mathrm{~nm} / \mathrm{s}$. The thickness of the layers is controlled by using a quartz-crystal monitor in this work. The current density-voltage $(J-V)$ and luminancevoltage $(L-V)$ characteristics of the devices were measured by using a Keithley 2400 (Keithley Instruments, Inc., USA) and a PR-655 (Photo Research, Inc., USA), respectively. The absorption spectrum was measured by using a U-3900 spectrophotometer (Hitachi, Japan). The photoluminescence (PL) spectrum and scanning electron microscope (SEM) images were measured by using an IK series (Kimmon Koha,

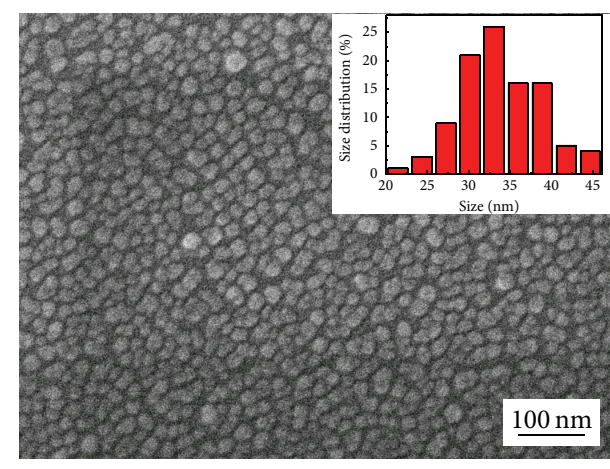

(a)

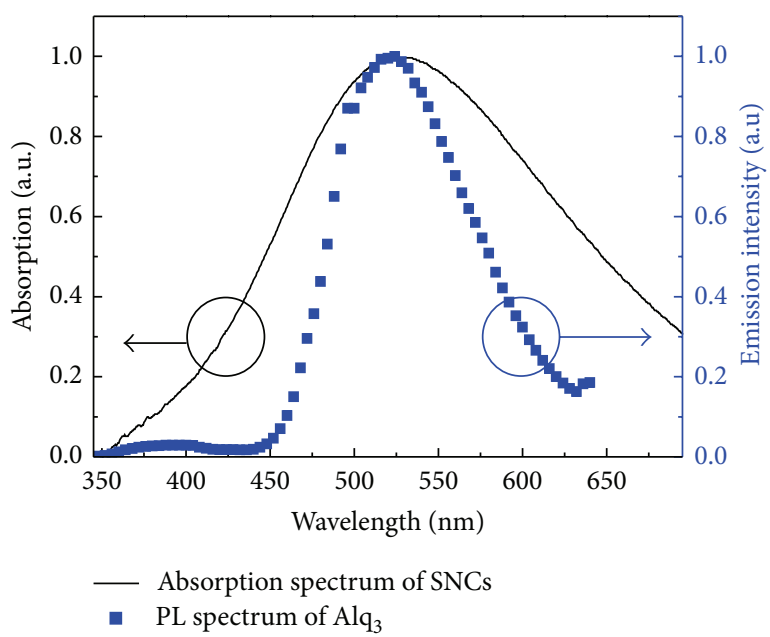

(b)

FIgURE 2: (a) SEM image of the SNPs on the glass surface. (b) Absorption spectrum of the SNPs and PL spectrum of the $\mathrm{Alq}_{3}$.

Japan) and a JSM-6700F (JEOL, Japan). All measurements were made at room temperature under ambient air.

\section{Results and Discussion}

Figure 2(a) shows the SEM image of SNPs on a glass substrate. The mean particle size of SNPs in Figure 2(a) was calculated and found to be $34 \mathrm{~nm}$. In addition, the size distribution of the SNCs is plotted in the inset of Figure 2(a). It is observed that the size distribution of the SNCs is from 21 to $45 \mathrm{~nm}$. The absorption spectrum of SNPs with a mean particle size of $34 \mathrm{~nm}$ and the PL spectrum of $\mathrm{Alq}_{3}$ are shown in Figure 2(b). It is observed that the absorption spectrum of SNPs with a mean particle size of $34 \mathrm{~nm}$ is about $525 \mathrm{~nm}$ and the PL spectrum of $\mathrm{Alq}_{3}$ is about $523 \mathrm{~nm}$. Furthermore, it can be seen that there is a significant overlap between the absorption spectrum of the SNPs and PL spectrum of $\mathrm{Alq}_{3}$. It is well known that the excellent overlap between the two spectra suggests that they can be used for SP-exciton coupling. In other words, this overlap implied that these SNPs can be used to increase the characters of OLEDs. 


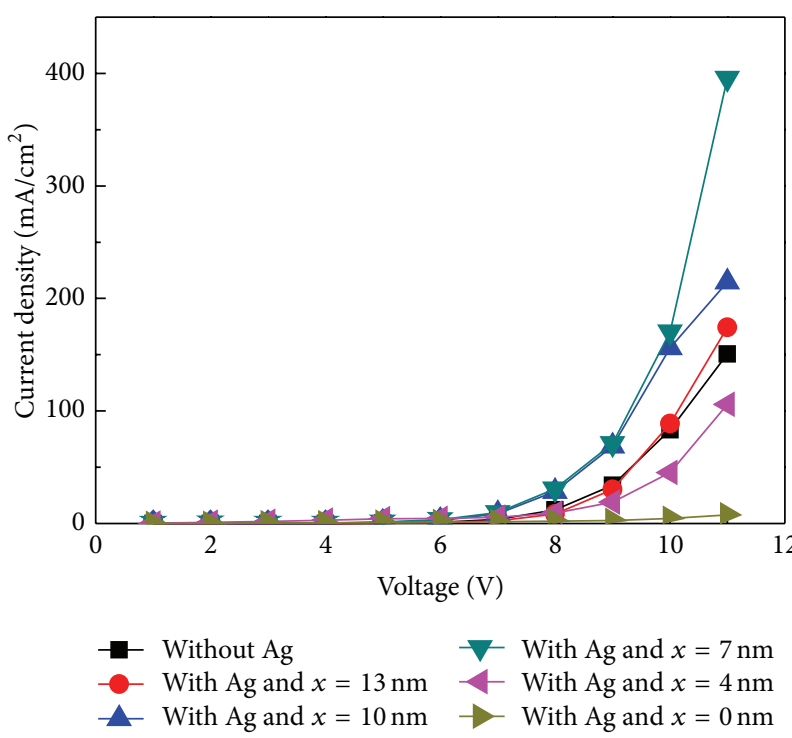

(a)

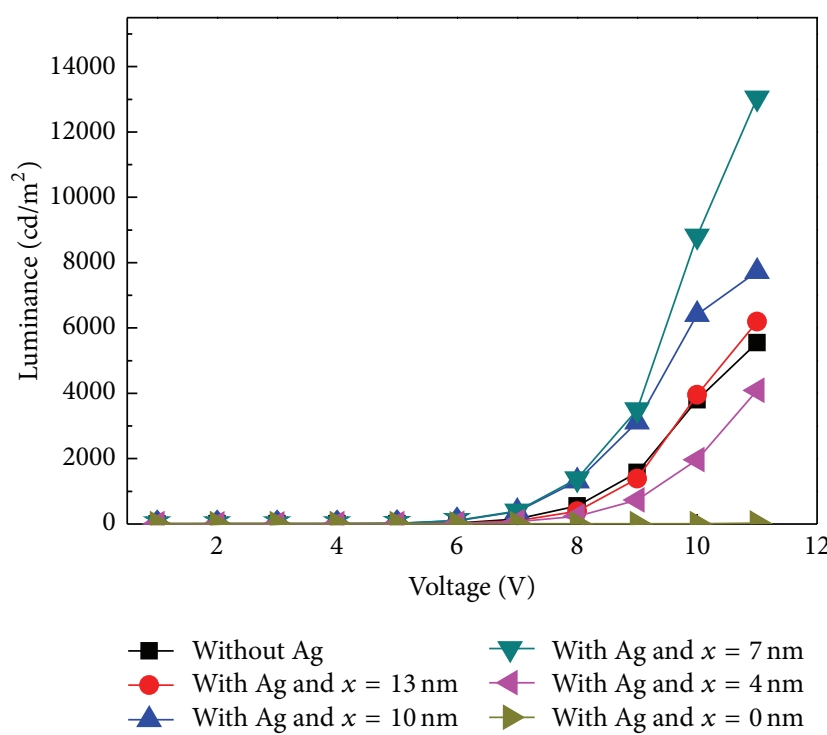

(b)

FIGURE 3: (a) Current density-voltage curves and (b) luminance-voltage curves of OLEDs with and without SNPs.

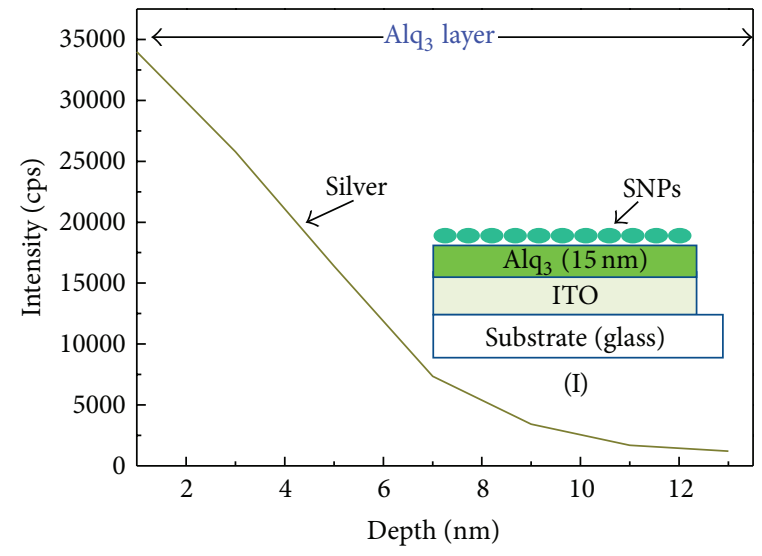

(a)

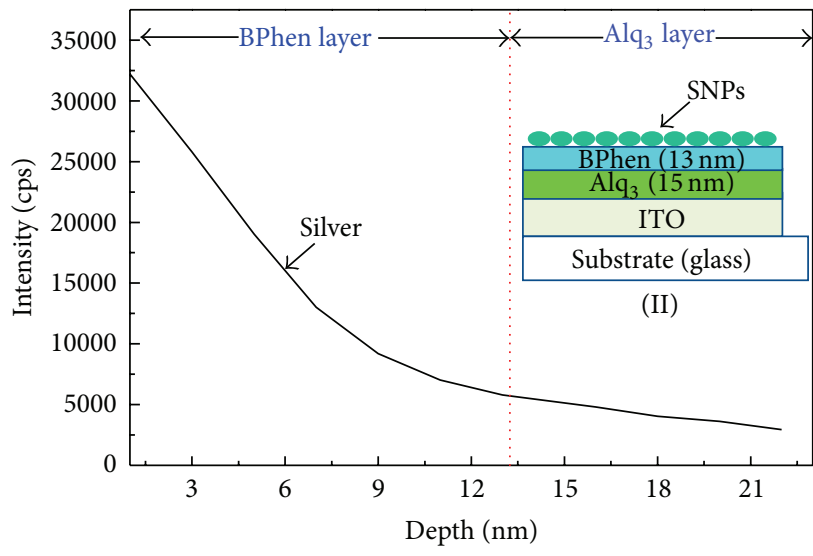

(b)

FIGURE 4: XPS depth profile of silver from the (a) structure I and (b) structure II.

The distance between the fluorescent molecule and the SNPs is a very important parameter in terms of the coupling between the SPs and excitons. Therefore, the distance was tuned by changing the thickness of the spacing layer (BPhen). The $J-V$ curves of the devices without SNPs and with different spacing distances between EML and SNPs are plotted in Figure 3(a). It can be clearly seen that the current density of the devices with SNPs at thickness of spacing $\geqq 7 \mathrm{~nm}$ is higher than that of devices without SNPs because the localized electric field around the SNPs is enhanced, resulting in an increase in electron injection from cathode electrode. Figure 3(b) shows the $L-V$ characteristic of the devices without SNPs and with different spacing distances between EML and SNPs. It is observed that the luminance of the devices was increased with a decrease in thickness of the spacing layer, in the condition of thickness of spacing $\geqq 7 \mathrm{~nm}$, because the surface-plasmon-enhanced spontaneous emission rate of SNPs is enhanced when the SNPs are close to the EML. In addition, the luminance of the device without a spacing layer is quite low compared with that of the device without SNPs, because the SNPs will penetrate considerably into EML.

To further confirm the relationship between the penetration of the SNPs and the luminance of the device, the X-ray photoelectron spectrometry (XPS) depth profile of silver is measured in this paper. The samples for analysis of XPS depth profile are prepared as follows: (I) ITO/Alq $\mathrm{I}_{3}(15 \mathrm{~nm}) / \mathrm{SNPs}$ and (II) ITO/Alq 3 (15 nm)/BPhen $(13 \mathrm{~nm}) / \mathrm{SNPs}$. Figures 4(a) and 4(b) show the XPS depth profile of silver from structures I and II, respectively. Structures I and II are plotted in the inset of Figures 3(a) and 3(b), respectively. It can be clearly seen that the intensity of the SNPs into the EML of the structure II is quite low, compared with that of structure I because structure II has a spacing layer which can impede 


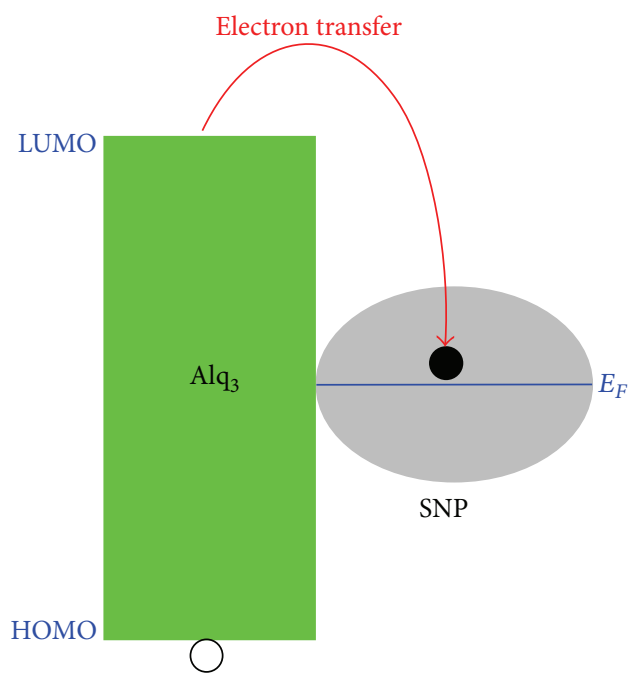

FIGURE 5: Schematic diagram of emission quenching process.

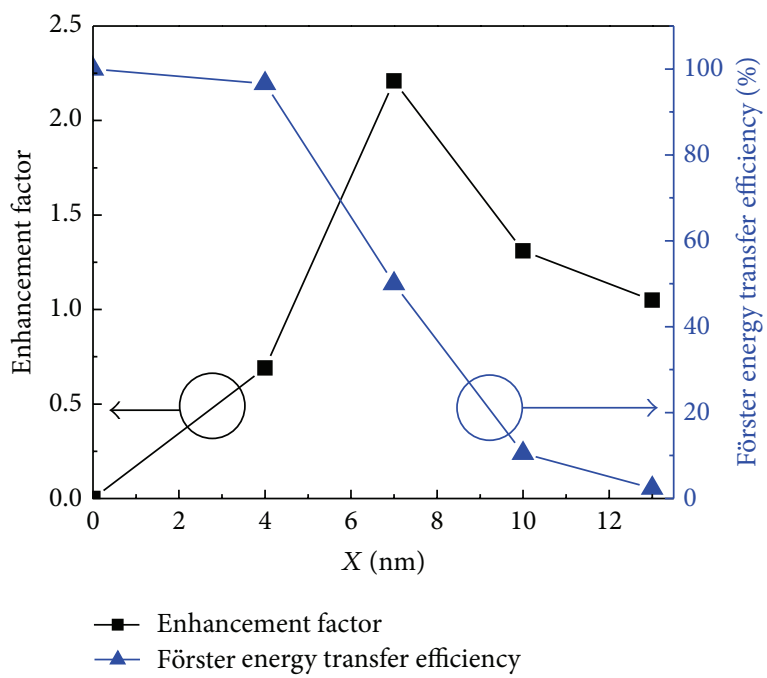

FIGURE 6: Enhancement factor and Förster energy transfer efficiency for different spacing distances between EML and SNPs.

the SNPs penetration into EML. Moreover, the emission quenching was caused when the SNPs penetrated into EML. The schematic diagram of the emission quenching process is shown in Figure 5. The emission quenching mainly resulted from the electron transfer from the lowest unoccupied molecular orbital (LUMO) of $\mathrm{Alq}_{3}$ to the Fermi level of the SNPs. Furthermore, the exciton is unable to be generated when the electron transfers from the LUMO of $\mathrm{Alq}_{3}$ to the Fermi level of the SNPs [15]. Therefore, when the SNPs penetrated into $\mathrm{Alq}_{3}$, the emission quenching of the device was caused. The enhancement and quenching of the device are a consequence of the interplay between the increase in radiative intensity by SPRE of SNPs and the emission quenching by electron transfer from the $\mathrm{Alq}_{3}$ to the SNPs.

Figure 6 shows the plots of the enhancement factor and the Förster energy transfer efficiency as a function of the spacing distance. The enhancement factor is expressed as $L_{2} / L_{1}$, where $L_{2}$ is the maximum luminance of the device with SNPs and $L_{1}$ is the maximum luminance of the device without SNPs. It is observed that the enhancement factors were increased with a decrease in thickness of the spacing layer, in the condition of thickness of spacing $\geqq 7 \mathrm{~nm}$ because the surface-plasmon-enhanced spontaneous emission rate of SNPs is enhanced when the SNPs are close to the EML. Furthermore, when the SNPs are close to the fluorescent molecule, the localized electric field around the SNPs is enhanced, resulting in an increase in electron injection from cathode electrode. In addition, the enhancement factors were decreased with a decrease in thickness of the spacing layer, in the condition of thickness of spacing $<7 \mathrm{~nm}$ because the amounts of SNPs penetration into EML are of considerable enhancement, resulting in the emission quenching of the device. It is also observed that the maximum enhancement factor is obtained when the Förster energy transfer efficiency is $50 \%$. The relationship between the Förster energy transfer efficiency and the spacing distance can be expressed as follows [16]:

$$
E=\frac{1}{1+\left(R / R_{0}\right)^{6}}
$$

where $E$ is the Förster energy transfer efficiency, $R$ is the spacing distance between the EML and SNPs, and $R_{0}$ is the Förster radius. The formula can clearly illustrate that the Förster energy transfer efficiency is enhanced with a decrease in the spacing distance between the EML and SNPs. Moreover, the Förster energy transfer efficiency of $50 \%$ is obtained when the spacing distance is equal to the Förster radius. The Förster radius can be expressed as follows [17]:

$$
R_{0}=0.211\left(\phi_{0} n^{-4} J k^{2}\right)^{1 / 6}
$$

where $R_{0}$ is the Förster radius, $\phi_{0}$ is the quantum yield of donor, $n$ is the refractive index of the medium, $J$ is the normalized overlap integral of the donor and the acceptor spectra, and $k$ is the orientation of the dipoles. Therefore, the Förster radius is calculated as $7 \mathrm{~nm}$ by using (2). In addition, the enhancement factor of the device without a spacing layer is quite low, compared with that of the device with a spacing layer, because the device without a spacing layer has an energy transfer of $100 \%$ from the fluorescent molecule to the SNPs, resulting in considerable nonradiative quenching of exciton. The schematic diagram of the nonradiative decay process of exciton is shown in Figure 7. The nonradiative decay process of exciton will be caused because of the excitation of surface plasmon polariton modes, which propagate along the surface of the SNPs. Moreover, the excitation of surface plasmon polariton modes results in an extra nonradiative decay channel at the interface between the fluorescent molecule and the SNPs [18]. Therefore, when the SNPs are very close to the fluorescent molecule, the SNPs will not only penetrate considerably into the EML but also generate an extra nonradiative decay channel. 


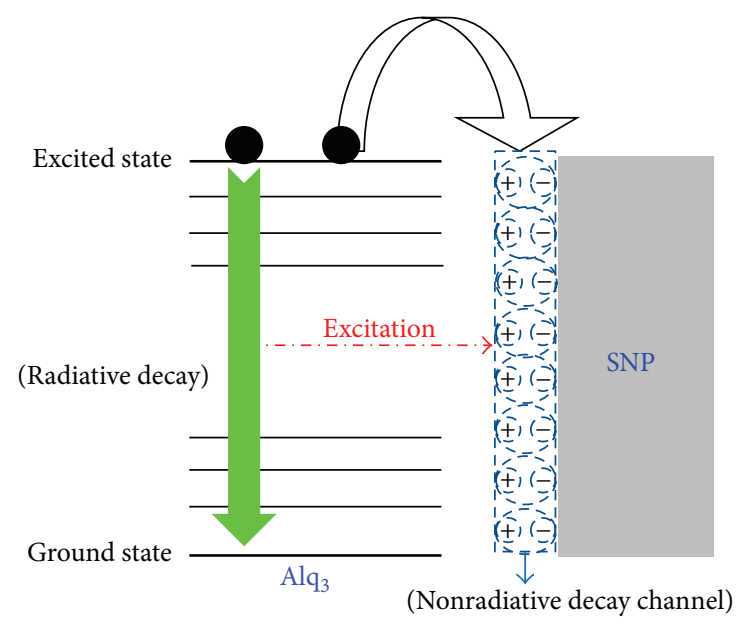

FIGURE 7: Schematic diagram of the nonradiative decay process of exciton.

\section{Conclusion}

In summary, the SNPs are introduced between the electrontransport layers, and their best performance is found to have the maximum luminance 2.4 times higher than the OLED without SNPs when the spacing distance is $7 \mathrm{~nm}$. When the SNPs are close to the fluorescent molecule, the SNPs will create two advantages: (i) they will enhance the spontaneous recombination of excitons due to the subtraction in the exciton lifetime and (ii) they will enhance the electron injection because of SPRE, which will induce the enhanced localized electric field around the SNPs. Nevertheless, when the SNPs are very close to the fluorescent molecule, the SNPs contrarily will result in two disadvantages: (i) they will penetrate considerably into the EML, resulting in the emission quenching of the device because the electron transfers from the LUMO of $\mathrm{Alq}_{3}$ to the Fermi level of the SNPs, and (ii) they will cause the nonradiative decay process of exciton because the excitation of surface plasmon polariton modes results in an extra nonradiative decay channel at the interface between the fluorescent molecule and the SNPs.

\section{Acknowledgment}

This work was partially supported by the National Science Council of Taiwan (NSCT) under Contract no. NSC102-2221E-390-019-MY2.

\section{References}

[1] Y. Zhao, L. Duan, D. Zhang et al., "Small molecular phosphorescent organic light-emitting diodes using a spin-coated hole blocking layer," Applied Physics Letters, vol. 100, no. 8, Article ID 083304, 4 pages, 2012.

[2] K. Narayan, S. Varadarajaperumal, G. Mohan Rao, M. Manor Varma, and T. Srinivas, "Effect of thickness variation of hole injection and hole blocking layers on the performance of fluorescent green organic light emitting diodes," Current Applied Physics, vol. 13, no. 1, pp. 18-25, 2013.
[3] J. Wang, J. Liu, S. Huang et al., "High efficiency green phosphorescent organic light-emitting diodes with a low roll-off at high brightness," Organic Electronics, vol. 14, no. 11, pp. 2854-2858, 2013.

[4] S. J. Lee, J. H. Seo, J. H. Kim et al., "Efficient triplet exciton confinement of white organic light-emitting diodes using a heavily doped phosphorescent blue emitter," Thin Solid Films, vol. 518, no. 22, pp. 6184-6187, 2010.

[5] Y. Zhang, M. Slootsky, and S. R. Forrest, "Enhanced efficiency in high-brightness fluorescent organic light emitting diodes through triplet management," Applied Physics Letters, vol. 99, no. 22, Article ID 223303, 3 pages, 2011.

[6] C. Yun, G. Xie, C. Murawski et al., "Understanding the influence of doping in efficient phosphorescent organic light-emitting diodes with an organic p-i-n homojunction," Organic Electronics, vol. 14, no. 7, pp. 1695-1703, 2013.

[7] Y. W. Park, J. H. Choi, T. H. Park et al., "Role of n-dopant based electron injection layer in $\mathrm{n}$-doped organic light-emitting diodes and its simple alternative," Applied Physics Letters, vol. 100, no. 1, Article ID 013312, 4 pages, 2012.

[8] K. Kim, K. Hong, I. Lee, S. Kim, and J. L. Lee, "Electron injection in magnesium-doped organic light-emitting diodes," Applied Physics Letters, vol. 101, no. 14, Article ID 141102, 4 pages, 2012.

[9] C.-Y. Cho, S.-J. Lee, J.-H. Song et al., "Enhanced optical output power of green light-emitting diodes by surface plasmon of gold nanoparticles," Applied Physics Letters, vol. 98, no. 5, Article ID 051106, 3 pages, 2011.

[10] F. Liu and J. M. Nunzi, "Enhanced organic light emitting diode and solar cell performances using silver nano-clusters," Organic Electronics, vol. 13, no. 9, pp. 1623-13632, 2012.

[11] Y. Xiao, J. P. Yang, P. P. Cheng et al., "Surface plasmonenhanced electroluminescence in organic light-emitting diodes incorporating Au nanoparticles," Applied Physics Letters, vol. 100, no. 1, Article ID 013308, 4 pages, 2012.

[12] K.-C. Tien, M.-S. Lin, Y.-H. Lin et al., "Utilizing surface plasmon polariton mediated energy transfer for tunable doubleemitting organic light-emitting devices," Organic Electronics, vol. 11, no. 3, pp. 397-406, 2010.

[13] W. L. Barnes, A. Dereux, and T. W. Ebbesen, "Surface plasmon subwavelength optics," Nature, vol. 424, no. 6950, pp. 824-830, 2003.

[14] A. Kumar, R. Srivastava, P. Tyagi, D. S. Mehta, and M. N. Kamalasanan, "Efficiency enhancement of organic light emitting diode via surface energy transfer between exciton and surface plasmon," Organic Electronics, vol. 13, no. 1, pp. 159-165, 2012.

[15] Y.-P. Hsieh, C.-T. Liang, Y.-F. Chen, C.-W. Lai, and P.-T. Chou, "Mechanism of giant enhancement of light emission from Au/ CdSe nanocomposites," Nanotechnology, vol. 18, no. 41, Article ID 415707, 4 pages, 2007.

[16] C. S. Yun, A. Javier, T. Jennings et al., "Nanometal surface energy transfer in optical rulers, breaking the FRET barrier," Journal of the American Chemical Society, vol. 127, no. 9, pp. 3115-3119, 2005.

[17] X. Zhang, C. A. Marocico, M. Lunz et al., "Wavelength, concentration, and distance dependence of nonradiative energy transfer to a plane of gold nanoparticles," ACS Nano, vol. 6, no. 10, pp. 9283-9290, 2012.

[18] G. W. Ford and W. H. Weber, "Electromagnetic interactions of molecules with metal surfaces," Physics Reports, vol. 113, no. 4, pp. 195-287, 1984. 

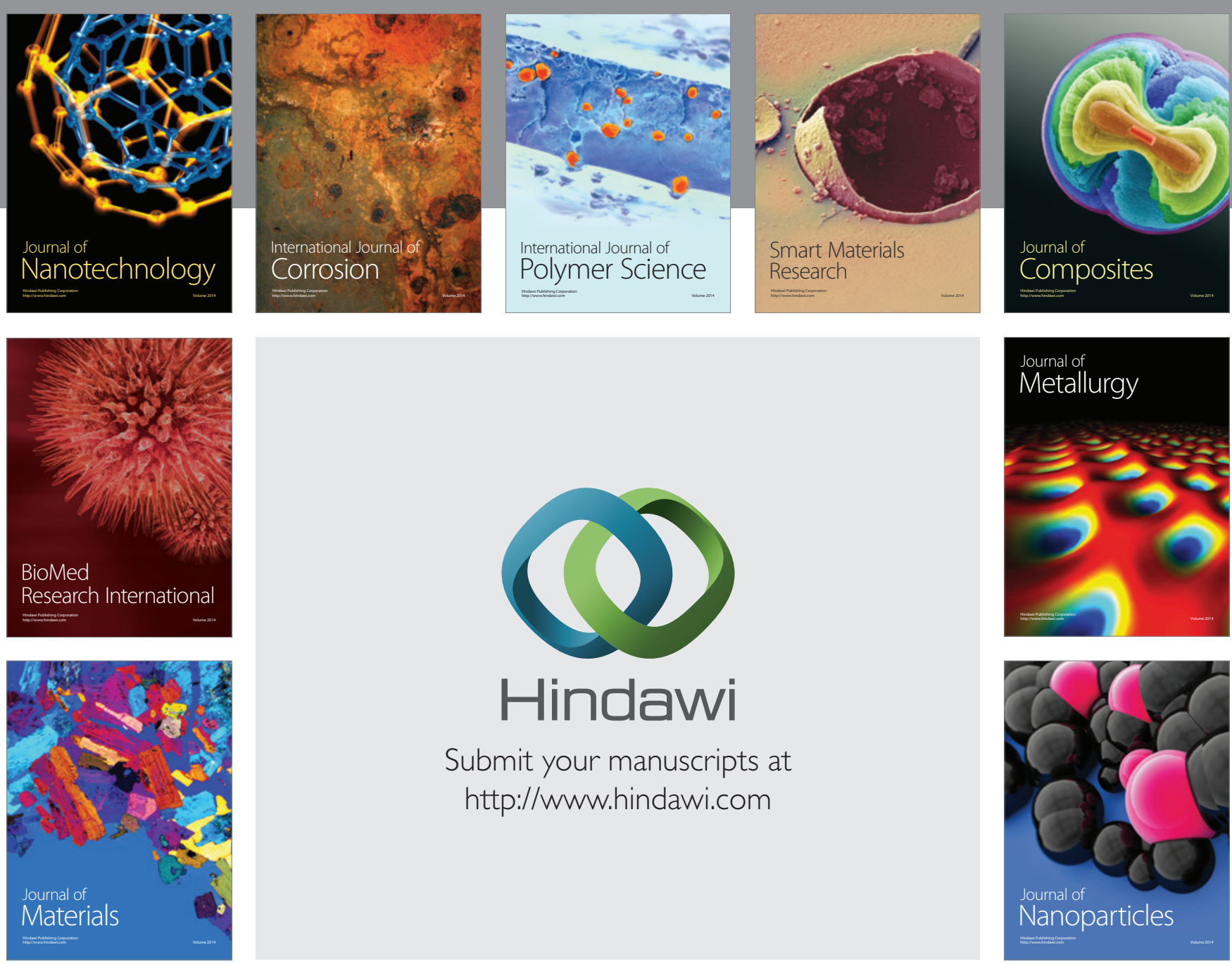

Submit your manuscripts at http://www.hindawi.com
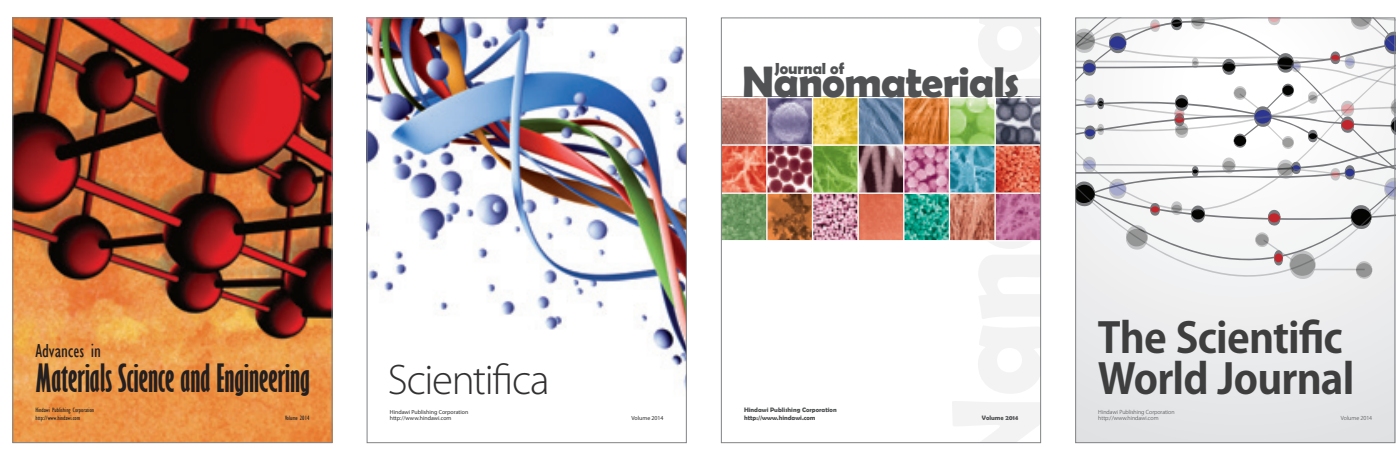

\section{The Scientific World Journal}
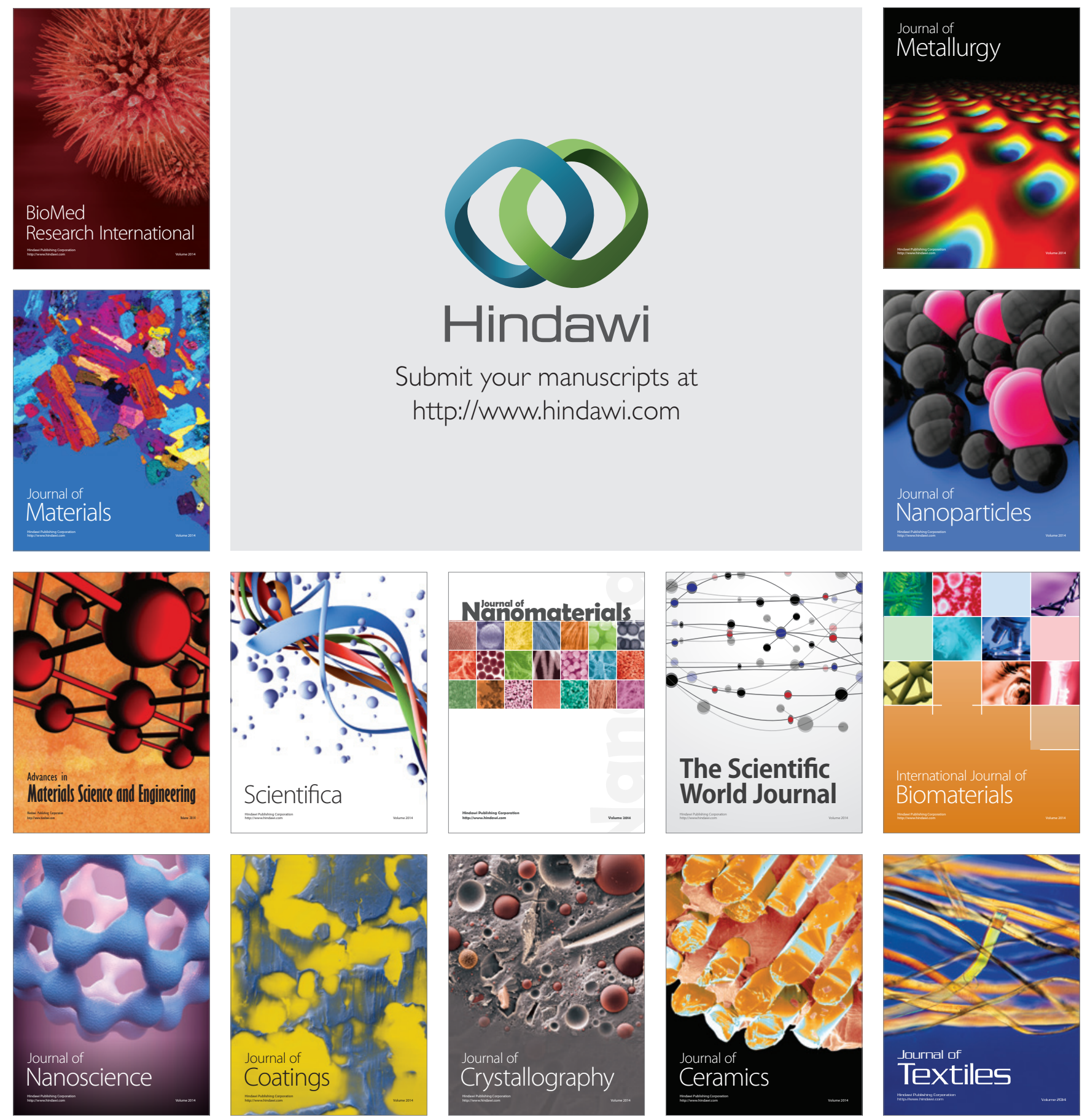